\title{
降水变化对内蒙古典型草原地上生物量的影响
}

\author{
苗百岭 1,2 梁存柱 ${ }^{*}$ 史亚博 ${ }^{1}$ 梁茂伟 ${ }^{1}$ 刘钟龄 1 \\ ${ }^{1}$ 内蒙古大学生态与环境学院, 呼和浩特 $010021 ;{ }^{2}$ 内蒙古自治区气象科学研究所, 呼和浩特 010051
}

摘 要 降水格局的变化是气候变化影响干旱和半干旱区草原植物群落结构和功能的关键过程。作为植物群落结构和功能的 基本组成单位——植物功能群——对气候变化的响应差异明显。有关不同植物功能群如何响应降水格局的研究, 有助于揭示 气候变化对干旱与半干旱区生态系统关键功能与过程稳定性的变异机制。该文利用1982-2015年的典型草原群落地上生物量 长期动态监测数据及其所对应的气候数据, 系统揭示降水变化对干旱与半干旱区生态系统生产力的影响。主要研究结果如下: 1) 1982-2015年内蒙古典型草原区降水变化特征明显, 主要表现在降水集中度呈显著的降低趋势; 小降水事件 $(\leqslant 5 \mathrm{~mm})$ 明显 增多。2)降水变化导致群落生物量呈下降趋势。其中, 多年生从生禾草的生物量呈上升趋势; 一二年生植物、多年生杂类草、 多年生根茎禾类草的生物量均呈下降趋势。3)群落生物量与生长季降水量、降水集中度呈显著的正相关关系。各功能群中, 多 年生杂类草、半灌木和生长季降水量呈显著正相关关系, 灌木与降水集中度呈显著负相关关系。4)群落生物量与各等级降水 的频率相关性均不显著, 但与 I-II类 $(0.1-10 \mathrm{~mm})$ 降水贡献率显著负相关。各功能群中, 多年生杂类草与 $\mathrm{I}$ 类降水 $(0.1-5.0 \mathrm{~mm})$ 的发生频率和降水贡献率均呈显著的负相关关系, 与VI类降水 $(20-25 \mathrm{~mm})$ 的发生频率和贡献率均呈显著的正相关关系。多年 生根茎禾类草和VIII类降水 $(>35 \mathrm{~mm})$ 的发生频率和贡献率均呈显著的正相关关系。说明小降水事件的增加将显著降低群落的 地上生物量, 小降水事件对干旱、半干旱区植被生长具有重要的生态学意义。

关键词 降水变化; 降水集中度; 典型草原; 地上生物量; 功能群

苗百岭, 梁存柱, 史亚博, 梁茂伟, 刘钟龄 (2019). 降水变化对内蒙古典型草原地上生物量的影响. 植物生态学报, 43, 557-565. DOI: $10.17521 /$ cjpe. 2018.0230

\section{Temporal changes in precipitation altered aboveground biomass in a typical steppe in Nei Mongol, China}

MIAO Bai-Ling ${ }^{1,2}$, LIANG Cun-Zhu $^{1 *}$, SHI Ya-Bo ${ }^{1}$, LIANG Mao-Wei ${ }^{1}$, and LIU Zhong-Ling ${ }^{1}$

${ }^{1}$ School of Ecology and Environment, Inner Mongolia University, Hohhot 010021, China; and ${ }^{2}$ Inner Mongolia Meteorological Institute, Hohhot 010051, China

\section{Abstract}

Aims Precipitation and its spatiotemporal changes are crucial for determining the effects of climate on plant community assembly and functioning of ecosystem (CAFE) in arid and semi-arid regions. Plant functional groups (PFGs) - an effective representation of CAFE-have been widely reported for their identity-dependent response to the changing climate. Here, we examine the responses of different PFGs to the temporal changes in precipitation by using aboveground biomass (AGB) as the dependent variable.

Methods We conducted a long-term ecological research of AGB since 1982 in a typical steppe grassland of Nei Mongol, China. We used the monthly-observation dataset from 1982 through 2015 to quantify the empirical relationships between AGB of different PDFs and precipitation.

Important findings We found that: 1) the decline in precipitation-concentration degree (PCD) was coupled with an increase in small rainfall events ( $\leqslant 5 \mathrm{~mm}$ ) during the 35-year study period; 2$)$ temporal changes in precipitation resulted in AGB decreases of annuals and biennials $(\mathrm{AB})$, perennial forbs (PF) and perennial rhizome grass (PR), whereas AGB increased for perennial bunchgrasses (PB); 3) AGB, PF and semi-shrubs (SS) were positively correlated with growing season rainfall with AGB positively correlated with PCD while AGB of the shrubs (S) was negatively correlated with PCD; 4) AGB showed no significant correlation with precipitation frequency, but a significant negative correlation for type I to II precipitation $0.1-10 \mathrm{~mm}$; 5) AGB had negative correlations with frequency and amount of type I $(0.1-5.0 \mathrm{~mm})$ and type VI $(20-25 \mathrm{~mm})$ precipitation. We concluded that the

收稿日期Received: 2018-09-18 接受日期Accepted: 2019-06-21

基金项目：国家重点研发计划(2016YFC0500503)和内蒙古自治区科技计划(20140409)。Supported by the National Key R\&D Program of China (2016YFC0500503), and the Science and Technology Project of Nei Mongol Autonomous Region (20140409).

* 通信作者Corresponding author (bilcz@imu.edu.cn) 
increase in small precipitation events will significantly reduce the AGB. These small precipitation events should be further explored for their ecological significances in the arid and semi-arid regions.

Key words precipitation dynamics; precipitation-concentration degree; typical steppe; aboveground biomass; plant functional groups

Miao BL, Liang CZ, Shi YB, Liang MW, Liu ZL (2019). Temporal changes in precipitation altered aboveground biomass in a typical steppe in Nei Mongol, China. Chinese Journal of Plant Ecology, 43, 557-565. DOI: 10.17521/cjpe.2018.0230

水分是干旱半干旱区植被生长发育的主要限制 因子(Sala et al., 1988; Easterling et al., 2000; Schwinning \& Sala, 2004), 并且能够影响陆地生态 系统对其他气候驱动因子变化的响应(Niu et al., 2007; Dijkstra et al., 2010), 而降水是这些地区的重 要水分获得途径。同时干旱半干旱地区的降水格局 也表现出较强的脉冲特征, 包括降水量的变化、降 水季节分布的变化, 以及降水间隔的变化(NoyMeir, 1973; Schwinning \& Sala, 2004; Schwinning et al., 2004)。许多大气环流模型都预测未来全球降水 格局会发生重大改变(IPCC, 2013), 同时还伴随着 降水间隔增大、小降水事件减少和极端降水事件增 加的趋势(Groisman et al., 1999; Easterling et al., 2000)。降水格局的变化不仅使陆地生态系统接受降 水的变幅增大, 还将从不同层次, 包括个体、群落、 生态系统水平, 对陆地生态系统产生影响(Knapp et al., 2001, 2002, 2008)。与其他陆地生态系统相比, 草原生态系统对降水变化更为敏感, 对资源可利用 性的瞬时波动响应更大(Knapp \& Smith, 2001; Conant et al., 2004), 在应对未来气候变化, 尤其是降 水变化时, 必须考虑草原生态系统结构与功能的重 要作用及其对气候变化的响应。

近些年来, 国内外学者对气候变化背景下草原 生态系统的植被生长等方面开展了大量的研究, 普 遍认为降水是影响植被生长和地上初级生产力的重 要因子(Knapp et al., 2017)。特别是对地处半干旱区 的草原生态系统, 降水是其主要水分来源, 对植被 生产力、生态系统结构和功能具有重要的影响 (Mowll et al., 2015), 在维持群落稳定方面起到重要 作用(Hallett et al., 2014), 如多数研究表明草原生态 系统地上净初级生产力与降水量存在线性正相关关 系(Knapp et al., 2001; Bai et al., 2008)等。另外, 不仅 降水量, 降水频度也可能对植被生产力有重要的影 响(Fay et al., 2003; Schwinning \& Sala, 2004; Knapp et al., 2008)。然而同一群落不同功能型的植物在长 期的进化过程中形成了各自的降水利用策略, 使得
它们对降水变化的响应也不尽相同(Robertson et al., $2009,2010)$ 。功能型、光合途径、形态生理特征和 生活史的不同使得不同物种对降水格局变化的响应 不同(Swemmer et al., 2007; Nippert et al., 2009)。为 了更好地理解群落对降水格局的响应, 我们应该比 较研究不同功能型植物之间对降水格局变化响应的 差别。

位于内蒙古锡林郭勒典型草原区的中国科学院 草原生态系统定位研究站羊草样地自1979年围封以 来一直未被利用, 可以认为群落生产力的波动是由 气候变化造成的(谭丽萍和周广胜, 2013)。该样地已 经连续观测了 35 年, 具有较长时间序列的群落调查 数据和气候观测数据, 能够反映降水变化和群落响 应的情况。本文利用1982-2015年群落组成、生物量 调查数据和相同时段的气候观测数据分析了近 30 年 来降水变化对草原生态系统地上生物量的影响。

\section{1 材料和方法}

\section{1 实验样地设置}

研究区位于内蒙古锡林郭勒草原自然保护区, 靠近中国科学院内蒙古草原生态系统定位研究站 (IMGERS, $43.63^{\circ} \mathrm{N}, 116.7^{\circ} \mathrm{E}$, 海拔1 $187 \mathrm{~m}$ )。该区 域是中亚半干早草原生态系统的一部分, 属大陆性 温带气候(Kawamura et al., 2005), 年平均温度为 0.7 ${ }^{\circ} \mathrm{C}$, 年降水量约为 $350 \mathrm{~mm}$, 主要集中在6-9月。植被 生长季从 4 月中旬开始, 到 9 月下旬结束, 生长期时 间相对较短 $(<150$ 天)。地带性植被类型以羊草 (Leymus chinensis)群落和大针茅(Stipa grandis)群落 为主(Tong et al., 2004)。土壤类型属于栗钙土, 土层 厚度为20-100 cm及以上, 伴有继发性的钻积层 (Wiesmeier et al., 2009)。

该羊草样地建于1979年, 面积为 $600 \mathrm{~m} \times 300 \mathrm{~m}$, 本研究资料为1982-2015年的样地观测数据, 以 10 年为周期(A01-A10)监测群落的地上生物量(图 1), 每年5-9月，以半个月为间隔做 1 次植被调查，共9期 监测数据(1-9)(图1), 每次监测 20 个分种样方, 登记 


\begin{tabular}{|c|}
\hline A10 \\
\hline A09 \\
\hline A08 \\
\hline A07 \\
\hline A06 \\
\hline A05 \\
\hline A04 \\
\hline A03 \\
\hline A02 \\
\hline A01 \\
\hline
\end{tabular}

图1内蒙古锡林郭勒典型草原生物量监测方法示意图。 A01-A10, 群落地上生物量逐年监测样带; 1-9, 每年生长季 生物量监测样地。

Fig. 1 Layout of the biomass sampling plots in a typical steppe grassland of Xilingol, Nei Mongol. A01-A10, a belt of biomass monitoring over a 10 -year period; $1-9$, a transect of biomass measurements during the growing seasons.

植物种类，测定植株高度、分种频度、分种密度、 分种地上生物量。同期气象资料来源于中国科学院 定位研究站(Li et al., 2015)。根据植物生活型将群落 分为多年生根茎禾类草 $(\mathrm{PR}) 、$ 多年生坐生禾草 $(\mathrm{PB}) 、$
多年生杂类草 $(\mathrm{PF})$ 、灌木 $(\mathrm{S})$ 、半灌木 $(\mathrm{SS})$ 和一二年 生植物(AB) 6 个功能群(Bai et al., 2004)(表1), 样地 具体信息见表2。

\section{2 降水等级划分标准}

通过分析历史数据, 并参考中国气象局降水等 级标准(高继卿等, 2015)以及Sala和Lauenroth (1982) 的研究结果, 本文将研究区降水分为以下 8 个等级 (表3)。其中, 将 $0.1 \leqslant R \leqslant 5 \mathrm{~mm}$ 定义为小降水事件, 并统计各等级降水的频率和贡献率。降水频率指各 等级降水日数占年降水日数百分比，降水贡献率指 各等级降水量占年降水量的比值。

降水变化可能仅仅是湿润土壤表面的小事件, 也可能是影响深层土壤水分动态、周期长达数十年 的大事件。有研究表明, $2 \mathrm{~mm}$ 的小降水事件只会引 起表层土壤微生物的活动, 导致土壤硝态氮增加或 发生短暂的分解变化, 大于 $3 \mathrm{~mm}$ 的降水可提高某 些高大植物的碳同化速率或者土壤结皮生物的净碳 收益, 而大于 $25 \mathrm{~mm}$ 的降水则可能引起多数荒漠植 物萌发。因此, 研究干旱地区降水脉冲量级特征和

表1 内蒙古锡林郭勒典型草原依据植物生活型划分的群落功能群

Table 1 Types of plant functional groups $\left(\mathrm{PFG}_{\mathrm{S}}\right)$ and associated life forms in the typical steppe grassland of Xilin Gol, Nei Mongol

\begin{tabular}{|c|c|}
\hline $\begin{array}{l}\text { 功能群 } \\
\text { Plant functional group }\end{array}$ & $\begin{array}{l}\text { 物种 } \\
\text { Species }\end{array}$ \\
\hline $\begin{array}{l}\text { 多年生根茎禾类草 } \\
\text { Perennial rhizome grass }\end{array}$ & 根茎冰草、黄囊薹草、羊草 Agropyron michnoi, Carex korshinskii, Leymus chinensis \\
\hline $\begin{array}{l}\text { 多年生丛生禾草 } \\
\text { Perennial bunchgrasses }\end{array}$ & $\begin{array}{l}\text { 䊁隐子草、大针茅、渐尖早熟禾、阿尔泰溚草、羊茅、羽茅 Cleistogenes squarrosa, Stipa grandis, Poa attenuata, Koeleria } \\
\text { macrantha, Festuca ovina, Achnatherum sibiricum }\end{array}$ \\
\hline $\begin{array}{l}\text { 多年生杂类草 } \\
\text { Perennial forbs }\end{array}$ & 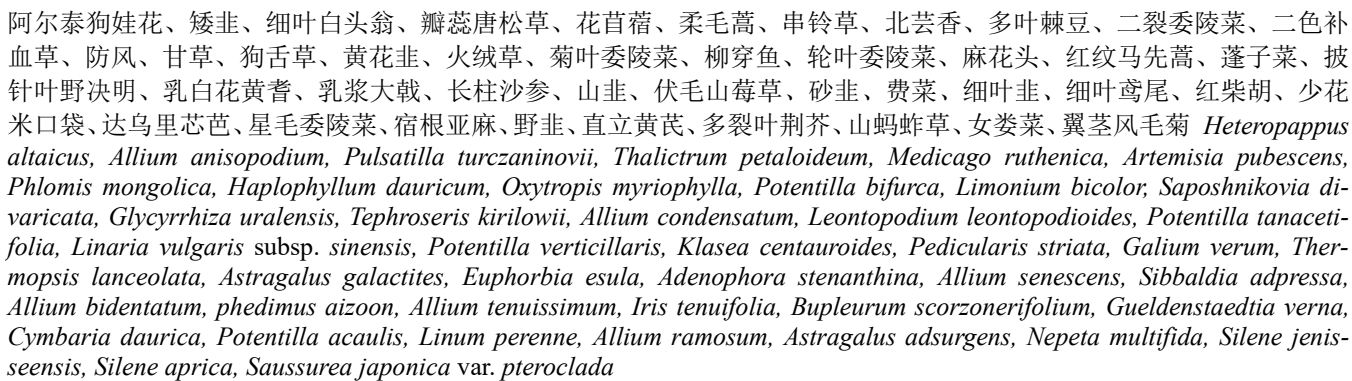 \\
\hline
\end{tabular}

灌木 Shrubs

半灌木 Semi-shrubs

二二年生植物

Annuals and biennials
小叶锦鸡儿 Caragana microphylla

冷蒿、木地肤、燥原荠 Artemisia frigida, Kochia prostrata, Ptilotricum canescens

刺藜、大籽蒿、瓦松、鹤蛗、猪毛蒿、藜、小花花旗杆、鳞叶龙胆、轴僽、猪毛菜 Dysphania aristata, Artemisia sieversiana, Orostachys fimbriatus, Lappula myosotis, Artemisia scoparia, Chenopodium album, Dontostemon micranthus, Gentiana squarrosa, Axyris amaranthoides, Salsola collina

表2 内蒙古锡林郭勒典型草原羊草样地的基本信息

Table 2 General information of Leymus chinensis site in the typical steppe grassland of Xilin Gol, Nei Mongol

\begin{tabular}{|c|c|c|c|c|c|c|c|c|}
\hline \multirow{2}{*}{$\begin{array}{l}\text { 地上生物量 } \\
\text { Aboveground } \\
\text { biomass }\left(\mathrm{g} \cdot \mathrm{m}^{-2}\right)\end{array}$} & \multicolumn{2}{|c|}{ 降水量 Precipitation (mm) } & \multicolumn{6}{|c|}{ 相对多度 Relative Abundance (\%) } \\
\hline & $\begin{array}{l}\text { 年 } \\
\text { Year }\end{array}$ & $\begin{array}{c}\text { 生长季 } \\
\text { Growing season }\end{array}$ & $\begin{array}{c}\text { 多年生根茎禾类草 } \\
\text { Perennial rhizome } \\
\text { grass }\end{array}$ & $\begin{array}{c}\text { 多年生丛生禾草 } \\
\text { Perennial } \\
\text { bunchgrasses }\end{array}$ & $\begin{array}{l}\text { 多年生杂类草 } \\
\text { Perennial forbs }\end{array}$ & $\begin{array}{c}\text { 一二年生植物 } \\
\text { Annuals and } \\
\text { biennials }\end{array}$ & $\begin{array}{c}\text { 灌木 } \\
\text { Shrubs }\end{array}$ & $\begin{array}{c}\text { 半灌木 } \\
\text { Semi-shrubs }\end{array}$ \\
\hline 195.8 & 330.8 & 287.9 & 63.4 & 19.3 & 9.9 & 4.8 & 1.9 & 0.7 \\
\hline
\end{tabular}


表3 内蒙古锡林郭勒典型草原羊草样地降水等级划分标准

Table 3 Precipitation level for type I-VIII at our study sites in the typical steppe grassland of Xilin Gol, Nei Mongol

\begin{tabular}{|c|c|c|}
\hline $\begin{array}{l}\text { 编号 } \\
\text { ID }\end{array}$ & $\begin{array}{c}\text { 日降水量 } \\
\text { Daily precipitation } \\
(\mathrm{mm})\end{array}$ & $\begin{array}{l}\text { 降水等级 } \\
\text { Precipitation grade }\end{array}$ \\
\hline I & $0.1-5$ & $\begin{array}{l}\text { 小雨/小雪-中雪 } \\
\text { Light rain/Light snow-Moderate snow }\end{array}$ \\
\hline II & $5-10$ & $\begin{array}{l}\text { 小雨/中雪-大雪 } \\
\text { Light rain/Moderate snow-Heavy snow }\end{array}$ \\
\hline III & $10-15$ & 中雨/暴雪 Moderate rain/Torrential snow \\
\hline IV & $15-20$ & 中雨/暴雪 Moderate rain/Torrential snow \\
\hline $\mathrm{V}$ & $20-25$ & $\begin{array}{l}\text { 中雨/暴雪-大暴雪 } \\
\text { Moderate rain/Torrential snow-Snowstorm }\end{array}$ \\
\hline VI & $25-30$ & 大雨/大暴雪 Heavy rain/Snowstorm \\
\hline VII & $30-35$ & 大雨/大暴雪 Heavy rain/Snowstorm \\
\hline VIII & $>35$ & $\begin{array}{l}\text { 大雨-暴雨/大暴雪-特大暴雪 } \\
\text { Heavy rain-Torrential rain/Snowstorm- } \\
\text { Historical extreme snow }\end{array}$ \\
\hline
\end{tabular}

变化趋势可更精细地了解荒漠植被与降水之间的关 系(刘冰等, 2010; 郑新倩等, 2012)。

\section{3 降水集中度计算方法}

为研究年内降水分配不均匀性特征, 或年内降 水分配情况，本文采用张录军和钱永甫(2004)定义 的表征单站降水量时间分配特征的参数，即降水集 中度 $(P C D)$, 其公式(张录军和钱永甫, 2004; 任志 艳等, 2016)可表示为:

$$
P C D_{i}=\sqrt{\left(\sum_{j=1}^{N} R_{i j} \times \sin \theta_{j}\right)^{2}+\left(\sum_{j=1}^{N} R_{i j} \times \cos \theta_{j}\right)^{2}} / R_{i}
$$

式中, $P C D_{i}$ 表示第 $i$ 年的降水集中度; $i$ 为年份; $j$ 为旬 序; $R_{i}$ 为第 $i$ 年内总降水量 $(\mathrm{mm}) ; R_{i j}$ 为第 $i$ 年第 $j$ 旬的降 水量 $(\mathrm{mm}) ; \theta_{j}=360^{\circ} \times(j-1) / 36$, 为研究时段内各
旬所对应的矢量角度。 $P C D_{i}$ 越接近于 1 , 则表示年降 水量集中在某一旬内; 越接近于 0 , 则表示年降水 量分布越均匀。

\section{2 结果}

\subsection{2-2015年研究区降水变化特征}

1982-2015年研究区年降水量总体变化趋势不 明显，有降低趋势( $\left.-8.44 \mathrm{~mm} \cdot 10 \mathrm{a}^{-1}, p=0.51\right)$, 但年 际间波动较大 $(21.84 \%)$ 。而降水集中度却呈极显著 降低趋势 $\left(-0.04 \cdot 10 \mathrm{a}^{-1}, p<0.001\right)$, 导致生长季的降 水也呈降低趋势 $\left(-14.27 \mathrm{~mm} \cdot 10 \mathrm{a}^{-1}, p=0.22\right)$, 降水 的年内分布格局已经发生了明显改变。

为了进一步分析降水格局的年内分布特征，对 各等级降水的频率和贡献率分别进行了统计分析 (表4)，结果表明：I类降水的平均发生频率 $(79.15 \%)$ 和贡献率 $(28.80 \%)$ 均为最高，随着降水等级的升高， 降水频率和贡献率逐渐降低, 变异系数逐渐加大, 降水波动性增强(VII类除外)。VI-VIII类降水出现了 43 次，其中VIII类降水就占了16次; 90年代出现最多, VIII类降水出现 10 次。年降水的波动主要是由 $>25 \mathrm{~mm}$ 的降水(VI-VIII类)变化引起的。

从变化趋势来看，1982-2015年I类降水的发生 频率和贡献率均有显著增加趋势 $(p \leqslant 0.001)$, II类 降水的发生频率呈显著降低趋势，但降水贡献率没 有明显变化趋势。小降水事件逐渐增多, 而5-10 $\mathrm{mm}$ 降水发生频率呈逐渐减少趋势(表4)。可见，小降 水事件发生的频率较高，对年降水总量的贡献率大 且稳定。

表4 1982-2015年内蒙古锡林郭勒典型草原羊草样地各等级降水变化特征

Table 4 Characteristics of precipitation changes for different levels in study area in the typical steppe grassland of Xilin Gol, Nei Mongol during 1982-2015

\begin{tabular}{|c|c|c|c|c|c|c|c|c|c|}
\hline \multirow{2}{*}{$\begin{array}{l}\text { 编号 } \\
\text { ID }\end{array}$} & \multirow{2}{*}{$\begin{array}{c}\text { 日降水量 }(\mathrm{mm}) \\
\text { Daily precipitation }\end{array}$} & \multicolumn{4}{|c|}{ 降水频率(\%) Precipitation frequency } & \multicolumn{4}{|c|}{ 降水贡献率(\%) Contribution to the total rainfall } \\
\hline & & $\begin{array}{l}\text { 平均值 } \\
\text { Average }\end{array}$ & $\begin{array}{c}\text { 变异系数 } \\
C V\end{array}$ & $\begin{array}{c}\text { 变化率 } \\
\text { Change rate }\end{array}$ & $p$ & $\begin{array}{l}\text { 平均值 } \\
\text { Average }\end{array}$ & $\begin{array}{c}\text { 变异系数 } \\
C V\end{array}$ & $\begin{array}{c}\text { 变化率 } \\
\text { Change rate }\end{array}$ & $p$ \\
\hline I & $0.1-5$ & 79.15 & 6.30 & $0.28^{* *}$ & 0.00 & 28.80 & 21.43 & $0.26^{*}$ & 0.02 \\
\hline II & $5-10$ & 10.62 & 40.81 & $-0.15^{*}$ & 0.04 & 21.71 & 44.11 & -0.07 & 0.66 \\
\hline III & $10-15$ & 5.27 & 49.04 & -0.06 & 0.21 & 18.12 & 45.72 & 0.00 & 0.99 \\
\hline IV & $15-20$ & 2.43 & 61.63 & -0.04 & 0.17 & 12.10 & 61.22 & -0.08 & 0.53 \\
\hline $\mathrm{V}$ & $20-25$ & 1.16 & 97.60 & -0.01 & 0.54 & 7.04 & 92.22 & -0.01 & 0.91 \\
\hline VI & $25-30$ & 0.52 & 121.14 & -0.01 & 0.24 & 3.67 & 118.56 & -0.07 & 0.40 \\
\hline VII & $30-35$ & 0.35 & 193.38 & -0.02 & 0.13 & 2.96 & 187.87 & -0.13 & 0.18 \\
\hline VIII & $>35$ & 0.49 & 171.51 & 0.01 & 0.63 & 5.60 & 163.85 & 0.11 & 0.50 \\
\hline
\end{tabular}

$*, p<0.05 ; * *, p<0.01 ; C V$, coefficient of variation. 
$2.21982-2015$ 年研究区群落地上生物量的变化 趋势

1982-2015年, 研究区群落生物量 $\left(B T_{\max }\right)$ (出现 在7-8月间)略呈下降趋势 $\left(-1.79 \mathrm{~g} \cdot \mathrm{m}^{-2} \cdot \mathrm{a}^{-1}, R^{2}=0.13\right.$, $p=0.04)$ 。其中, 多年生杂类草、多年生根茎禾类草、 灌木的生物量呈显著下降趋势 $(p \leqslant 0.04)$, 变化率 分别为每年 $-0.74 \mathrm{~g} \cdot \mathrm{m}^{-2} 、-1.15 \mathrm{~g} \cdot \mathrm{m}^{-2} 、-0.61 \mathrm{~g} \cdot \mathrm{m}^{-2}$; 多年生丛生禾草与一二年生植物的生物量变化趋势 不显著 $(p \geqslant 0.51)$, 斜率分别为 0.29 和 -0.02 ; 而半灌木 的生物量却呈显著上升趋势 $\left(0.44 \mathrm{~g} \cdot \mathrm{m}^{-2} \cdot \mathrm{a}^{-1}, p<0.001\right)$ 。

\section{3 降水变化对群落地上生物量的影响}

本文利用1982-2015年群落、各功能型地上生物 量与生长季降水量、降水集中度进行了回归分析。 分析结果发现, 群落地上生物量与生长季降水量 $(R)$ 、降水集中度呈显著的正相关关系, 随着生长季 降水量的减少和降水集中度的降低, 地上生物量略 有降低趋势(图2A、2B)。其回归方程分别为: $B T_{\text {max }}=$ $0.27 R+112.06\left(R^{2}=0.17, p=0.02\right) ; B T_{\max }=$ $177.33 P C D+76.25\left(R^{2}=0.12, p=0.05\right)$ 。

在各功能群中, 多年生杂类草的地上生物量和 生长季降水量显著正相关 $\left(B T_{\mathrm{PF}}=0.15 R-14.86\right.$,
$\left.R^{2}=0.27, p<0.01\right)$, 半灌木的地上生物量与降水集 中度显著负相关 $\left(B T_{\mathrm{SS}}=-33.79 P C D+30.65, R^{2}=\right.$ $0.16, p=0.02)$ 。随着研究区降水量的减少和集中度 的降低, 多年生杂类草的地上生物量有减少趋势, 而半灌木有明显增加趋势。除此以外, 其他各功能 群的地上生物量与生长季降水量和降水集中度的相 关性均不显著, 对降水变化的响应较弱。

为了探讨不同等级降水事件对群落的影响程度, 本文还利用了1982-2015年不同等级降水的发生频 率和贡献率分别与群落、各功能群的地上生物量进 行了相关分析(表5, 表6)。从表5中可知, $B T_{\max }$ 与各 等级降水的频率相关性均不显著。各功能群中, 多 年生杂类草与 I类降水 $(0.1-5 \mathrm{~mm})$ 发生频率呈显著 的负相关关系, 与VI类降水 (20-25 mm) 发生频率呈 显著的正相关关系。多年生根茎禾类草和VIII类降 水(>35 mm)发生频率显著正相关。

从表6中可知, 只有 $\mathrm{I}-\mathrm{II}$ 类降水贡献率与群落 $B T_{\max }$ 呈显著的负相关关系 $(p<0.048)$, 其他等级降 水贡献率与群落生物量相关性均不显著 $(p>$ $0.160)$ 。在各功能群中, 多年生杂类草与 I类降水贡 献率呈显著的负相关关系, 与VI类降水贡献率呈显
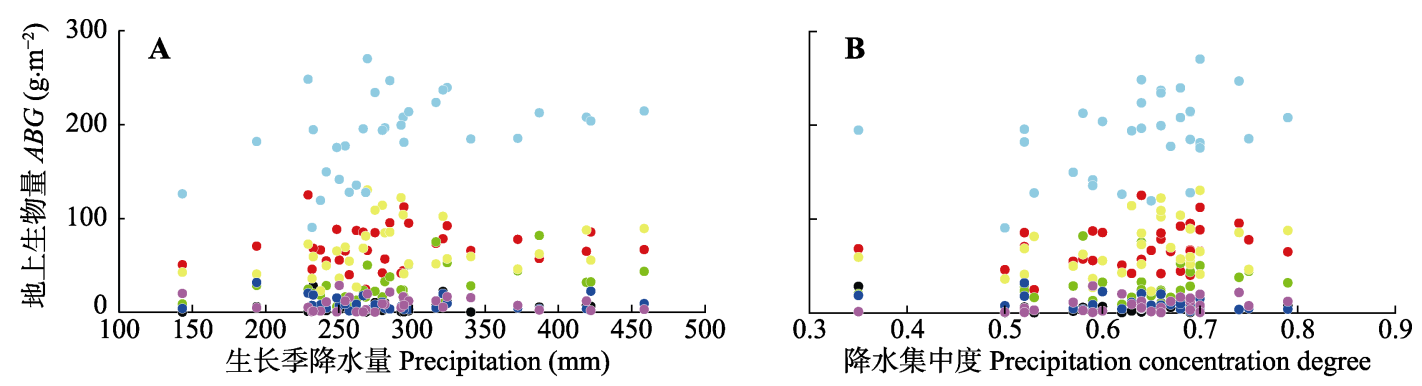

- 一二年生植物 Annuals and biennials - 多年生丛生禾草 Perennial bunchgrasses - 多年生杂类草 Perennial forbs 多年生根茎禾类草 Perennial rhizome grass ・半灌木 Semi-Shrubs ・灌木 Shrubs ・群落 Community

图2 内蒙古锡林郭勒典型草原羊草样地群落和各功能群的地上生物量对降水变化的响应。

Fig. 2 Response of aboveground biomass $(A G B)$ by community and functional group to precipitation in the typical grassland of Xilin Gol, Nei Mongol.

表5 内蒙古锡林郭勒典型草原羊草样地群落地上峰值生物量与各等级降水发生频率之间的相关分析

Table 5 Correlation between peak biomass and the frequency of precipitation in different levels in the typical grassland of Xilin Gol, Nei Mongol

\begin{tabular}{|c|c|c|c|c|c|c|c|c|}
\hline $\begin{array}{l}\text { 编号 } \\
\text { ID }\end{array}$ & $\begin{array}{c}\text { 日降水量(mm) } \\
\text { Daily precipitation }\end{array}$ & $\begin{array}{l}\text { 生物量 } \\
\text { Biomass }\end{array}$ & $\begin{array}{l}\text { 多年生杂类草 } \\
\text { Perennial forbs }\end{array}$ & $\begin{array}{c}\text { 多年生根茎禾草 } \\
\text { Perennial rhizome } \\
\text { grass }\end{array}$ & $\begin{array}{c}\text { 多年生丛生禾草 } \\
\text { Perennial } \\
\text { bunchgrasses }\end{array}$ & $\begin{array}{c}\text { 一二年生植物 } \\
\text { Annuals and } \\
\text { biennials }\end{array}$ & $\begin{array}{c}\text { 半灌木 } \\
\text { Semi-shrubs }\end{array}$ & $\begin{array}{l}\text { 灌木 } \\
\text { Shrubs }\end{array}$ \\
\hline I & $0.1-5$ & -0.23 & $-0.50^{* *}$ & -0.02 & -0.04 & -0.01 & $0.40^{*}$ & -0.27 \\
\hline II & $5-10$ & -0.06 & 0.19 & -0.13 & -0.14 & 0.15 & -0.28 & 0.18 \\
\hline III & $10-15$ & 0.15 & 0.25 & -0.01 & 0.12 & -0.13 & -0.16 & 0.23 \\
\hline IV & $15-20$ & 0.24 & 0.24 & 0.04 & 0.18 & -0.05 & -0.03 & 0.17 \\
\hline V & $20-25$ & 0.12 & 0.10 & 0.07 & 0.17 & -0.09 & -0.08 & -0.13 \\
\hline VI & $25-30$ & 0.33 & $0.55^{* *}$ & 0.19 & 0.04 & -0.04 & -0.17 & -0.08 \\
\hline VII & $30-35$ & 0.19 & 0.34 & 0.11 & -0.02 & -0.07 & -0.19 & 0.11 \\
\hline VIII & $>35$ & 0.22 & -0.00 & $0.39^{*}$ & -0.00 & -0.00 & -0.00 & -0.17 \\
\hline
\end{tabular}


表6 内蒙古锡林郭勒典型草原羊草样地群落地上生物量与各等级降水贡献率之间的相关分析

Table 6 Correlation analysis between peak biomass and contribution rate of precipitation in different grades in the typical grassland of Xilin Gol, Nei Mongol

\begin{tabular}{|c|c|c|c|c|c|c|c|c|}
\hline $\begin{array}{l}\text { 编号 } \\
\text { ID }\end{array}$ & $\begin{array}{c}\text { 日降水量(mm) } \\
\text { Daily precipitation }\end{array}$ & $\begin{array}{l}\text { 生物量 } \\
\text { Biomass }\end{array}$ & $\begin{array}{l}\text { 多年生杂类草 } \\
\text { Perennial forbs }\end{array}$ & $\begin{array}{c}\text { 多年生根茎禾草 } \\
\text { Perennial rhizome } \\
\text { grass }\end{array}$ & $\begin{array}{c}\text { 多年生从生禾草 } \\
\text { Perennial } \\
\text { bunchgrasses }\end{array}$ & $\begin{array}{c}\text { 一二年生植物 } \\
\text { Annuals and } \\
\text { biennials }\end{array}$ & $\begin{array}{c}\text { 半灌木 } \\
\text { Semi-shrubs }\end{array}$ & $\begin{array}{c}\text { 灌木 } \\
\text { Shrubs }\end{array}$ \\
\hline I & $0.1-5$ & $-0.53^{* *}$ & $-0.58^{* *}$ & -0.28 & -0.22 & -0.02 & 0.23 & -0.07 \\
\hline II & $5-10$ & -0.30 & -0.08 & -0.30 & -0.21 & 0.21 & -0.08 & 0.10 \\
\hline III & $10-15$ & 0.00 & 0.04 & -0.06 & 0.03 & -0.15 & 0.01 & 0.16 \\
\hline IV & $15-20$ & 0.17 & 0.10 & -0.00 & 0.18 & -0.02 & 0.12 & 0.13 \\
\hline $\mathrm{V}$ & $20-25$ & 0.06 & -0.02 & 0.07 & 0.14 & -0.09 & -0.03 & -0.19 \\
\hline VI & $25-30$ & 0.26 & $0.50^{* *}$ & 0.10 & 0.06 & -0.02 & -0.15 & -0.11 \\
\hline VII & $30-35$ & 0.20 & 0.32 & 0.12 & 0.01 & -0.06 & -0.15 & 0.08 \\
\hline VIII & $>35$ & 0.24 & -0.05 & $0.38 *$ & 0.06 & 0.06 & 0.01 & -0.18 \\
\hline
\end{tabular}

$*, p<0.05 ; * *, p<0.01$ 。

著的正相关关系。多年生根茎禾类草与VIII类降水 贡献率呈显著的正相关关系。

\section{3 讨论}

\section{1 研究区降水变化对群落地上生物量的影响}

在干旱与半干旱区, 降水事件通常以脉动的形 式发生，降水发生时间、持续时间、强度等参数具 有较大的变异性(Sala \& Lauenroth, 1982; 赵学勇等, 2014)。本文发现：1982-2015年研究区 $\leqslant 5 \mathrm{~mm}$ 和 $\leqslant 10 \mathrm{~mm}$ 降水分别占总降水频次的 $80 \%$ 和 $90 \%$ ，与 科尔沁沙地的统计结果(马文武花等, 2015)一致, 也与 Sala和Lauenroth (1982)在北美草原的统计结果接 近。近些年来, 研究区小降水事件的发生频率和降 水贡献率都有逐年增加的趋势, 直接导致了降水集 中度的降低。

以往研究发现，降水脉动可以导致土壤水分与 养分等资源也呈脉动状态，进而影响着种群动态、 群落变化等生态系统过程(Noy-Meir，1973)。NoyMeir (1973)发现降水脉动可能是干旱与半干旱生态 系统演替的核心驱动力和不同植物功能类型共存的 重要原因。国内外学者围绕降水脉动发生时间、强 度及其对生态系统的影响等方面, 先后提出了脉动存咜理论(Pulse-Reserve)(Noy-Meir，1973)、水分分 割理论(Two-Layer Soil Water-Partitioning)(Walter, 1971)等理论模型。其中, 脉动-存伧理论是干旱与半 干旱区生态系统研究中广泛应用的一个理论。该理 论认为降水脉动能够触发植物生长脉动与碳和能量 储存之间的关系，这种存咜能力在两次降水间隔期 内处于休眠状态, 此时生态系统初级生产力不再增 长(Noy-Meir, 1973; Schwinning et al., 2004; 赵文智
和刘鹄，2011)。Ludwig等(1996)认为该理论体现了 干旱与半干旱降水事件的生态学意义。但是 Reynolds等(2004)认为, 在生态系统中, 大降水事件 不一定使生态系统生产力出现净增长(Snyder et al., 2004), 而小降水事件却有可能导致明显的生态响 应，降水与生态系统之间的关系并不完全符合脉动存咜理论(Reynolds et al., 2000)。

在许多研究中, $\leqslant 5 \mathrm{~mm}$ 的降水被认为是无效降 水, 因其仅能润湿表层土壤, 不能到达植物根系 (Dougherty et al., 1996)。Owens等( 2006)研究发现, $\leqslant 2.5 \mathrm{~mm}$ 的降水几乎全部被植物冠层截留。通常认 为, 被冠层截留的这部分降水以蒸发形式返回大气, 而不能被根系吸收利用(Owens et al., 2006)。本文中 群落生物量与 $\leqslant 5 \mathrm{~mm}$ 的降水均呈显著的负相关关 系，与生长季降水量呈显著正相关关系。和生长季 降水对群落地上生物量有促进作用不同，小降水事 件对群落地上生物量有明显的抑制作用，可能是因 为小于 $10 \mathrm{~mm}$ 的降水在土壤里下渗较浅, 大部分水 分将会被蒸发掉, 对土壤的补给较少, 能被植物利 用的更少。郭柯等(2000)在毛乌素沙地的研究也表 明, 小于 $5 \mathrm{~mm}$ 的降水在固定沙地下渗的深度只有 5 $\mathrm{cm}$ 左右，雨后绝大部分的雨水被蒸发掉，对固定沙 地灌木根系层土壤水分的补充基本没有效果。本研 究进一步发现：在过去的30多年间，研究区小降水 事件 $(0.1-5 \mathrm{~mm})$ 的发生频率和降水贡献率都呈逐年 增加趋势, 导致了降水集中度下降, 造成 $B T_{\max }$ 略有 下降趋势 $(p=0.051)$ 。综合来看, 小降水事件主要通 过影响降水的分布和强度, 间接影响土壤水分, 进 而对生态系统的功能造成影响，这与Reynolds等 (2004)在北美的研究结论基本一致。Lauenroth和Sala 
(1992)在科罗拉多的短草草原研究发现，15-30 mm 的降水是 $A N P P$ 受影响的有效降水。本研究中发现, $\geqslant 25 \mathrm{~mm}$ 的降水与多年生根茎禾类草、多年生杂类 草的地上生物量显著正相关, 能够增加群落的地上 生物量, 是有效降水。

\section{2 不同生活型植物对降水变化的响应}

本研究发现, 小降水事件(I)的增多降低了群落 中多年生杂类草的地上生物量, 大降水事件(VIVIII)的波动会对多年生根茎禾类草和多年生杂类 草的地上生物量产生显著影响, 而降水变化对其他 几个植物功能型均无显著影响, 这可能与不同植物 功能型获得水资源的策略不同有关。Reynolds等 (2004)认为植物的这种响应过程与群落组成、前期 土壤水分状况和生长阶段都有密切关系。多年生从 生禾草与一二年生植物更多利用的是表层土壤水分, 它们的根系较浅, 大部分根系集中在土壤剖面的上 部(Sims et al., 1978), 能够对土壤水分的增加做出 非常迅速的反应。相反, 多年生杂类草与多年生根 茎禾类草等植物根系相对较深, 利用的主要是深层 土壤水分(Soriano \& Sala, 1984)。Walter的水分分割 理论认为, 小降水事件能有效地补给表层土壤水分, 而大降水事件则能补给深层土壤水分。随着研究区 小降水事件的增多, 对深层土壤水分的补给越来越 少, 从而对多年生杂类草和多年生根茎禾类草等深 根系植物产生显著影响, 降水格局变化导致土壤水 分变异性增加引起根系活性降低, 这可能是导致多 年生杂类草生物量降低的一个机制。另外, 禾本科 $\mathrm{C}_{4}$ 植物比多年生杂类草 $\mathrm{C}_{3}$ 植物的光合水分利用效率 更高(Knapp \& Medina, 1999), 能够有效抵御降水变 化的影响(Fay et al., 2003; 牛书丽等, 2004), 这可能 是多年生丛生禾草等生物量相对稳定的一个机制。

羊草与大针茅种群是研究区的主要建群种, 尽 管它们广泛共生, 有着相似的光合生理和生活史, 但它们对降水的变化表现出截然不同的反应。本研 究中发现以羊草为代表的多年生根茎禾类草与 $\geqslant 35$ $\mathrm{mm}$ 的降水发生频率与贡献率均呈显著的正相关关 系, 而以大针茅为代表的多年生丛生禾草对水分梯 度的响应并不显著, 这可能与两种植物的根系构型 有关。鲍雅静等(2019)研究发现, 相比大针茅种群, 羊草根系特征对水分梯度的响应更为显著, 羊草主 要是通过根茎长度和密度等数量特征来调节对水分 梯度的响应, 而大针茅的根系对水分梯度的响应并
不敏感，可能把更多的能力投资在从幅的扩大上, 这与崔骁勇等(2001)的研究结论基本一致, 其认为 大针茅对干旱的调节机制不是通过调整根系提高水 分利用效率实现的, 而是通过提高水分竞争能力、 降低水分蒸腾来实现的。因此，相对于优势种，亚优 势种受降水变化的影响可能更强，正是因为应对降 水变化策略的差异性, 才使得草原生态系统保持稳 定。

\section{3 结论}

1) 1982-2015年, 研究区年降水量变化趋势均 不明显，而降水集中度呈显著的降低趋势。在各等 级降水事件中, $\leqslant 5 \mathrm{~mm}$ 降水事件的发生频率和对年 降水量的贡献率均有增加趋势，导致了降水集中度 的显著降低; $\geqslant 25 \mathrm{~mm}$ 降水事件和连续无降水日的 变化造成了降水的年际间波动。

2） 1982-2015年，研究区群落地上生物量没有 明显变化, 总生物量略显下降趋势。生长季降水的 波动是群落地上生物量的主要限制因素。近些年来, 降水事件组合发生的改变, 对群落地上生物量产生 了显著的影响。

\section{参考文献}

Bai YF, Han XG, Wu JG, Chen ZZ, Li LH (2004). Ecosystem stability and compensatory effects in the Inner Mongolia grassland. Nature, 431, 181-184.

Bai YF, Wu JG, Xing Q, Pan QM, Huang JH, Yang DL, Han XG (2008). Primary production and rain use efficiency across a precipitation gradient on the Mongolia Plateau. Ecology, 89, 2140-2153.

Bao YJ, Cao M, Li ZH, Guo P, Zhang J, Qin J (2019). A comparative study of the response of Leymus chinensis and Stipa grandis root characteristics to moisture gradients. Acta Ecologica Sinica, 39，1063-1070. [鲍雅静，曹明, 李政海, 郭鹏, 张靖, 秦洁 (2019). 羊草与大针茅根系 构型对水分梯度响应的比较研究. 生态学报, 39, 1063-1070.]

Conant RT, Dalla-Betta P, Klopatek CC, Klopatek JM (2004). Controls on soil respiration in semiarid soils. Soil Biology \& Biochemistry, 36, 945-951.

Cui XY, Chen ZZ, Du ZC (2001). Study on light- and wateruse characteristics of main plants in semiarid steppe. Acta Prataculturae Sinica, 10, 14-21. [崔骁勇, 陈佐忠, 杜占 池 (2001). 半干旱草原主要植物光能和水分利用特征 的研究. 草业学报, 10, 14-21.]

Dijkstra FA, Blumenthal D, Morgan JA, LeCain DR, Follett RF (2010). Elevated $\mathrm{CO}_{2}$ effects on semi-arid grassland plants in relation to water availability and competition. Functional 
Ecology, 24, 1152-1161.

Dougherty RL, Lauenroth WK, Singh JS (1996). Response of a grassland cactus to frequency and size of rainfall events in a North American shortgrass steppe. Journal of Ecology, 84, 177-183.

Easterling DR, Meehl GA, Parmesan C, Changnon SA, Karl TR, Mearns LO (2000). Climate extremes: Observations, modeling, and impacts. Science, 289, 2068-2074.

Fay PA, Carlisle JD, Knapp AK, Blair JM, Collins SL (2003). Productivity responses to altered rainfall patterns in a $\mathrm{C}_{4}$-dominated grassland. Oecologia, 137, 245-251.

Gao JQ, Yang XG, Dong CY, Li KN (2015). Precipitation resource changed characteristics in arid and humid regions in Northern China with climate changes. Transactions of the Chinese Society of Agricultural Engineering, 31, 99-110. [高继卿, 杨晓光, 董朝阳, 李克南 (2015). 气 候变化背景下中国北方干湿区降水资源变化特征分析. 农业工程学报, 31, 99-110.]

Groisman PY, Karl TR, Easterling DR, Knight RW, Jamason PF, Hennessy KJ, Suppiah R, Page CM, Wibig J, Fortuniak K, Razuvaev VN, Douglas A, Førland E, Zhai PM (1999). Changes in the probability of heavy precipitation: Important indicators of climatic change. Climatic Change, 42, 243-283.

Guo K, Dong XJ, Liu ZM (2000). Characteristics of soil moisture content on sand dunes in $\mathrm{Mu}$ Us sandy grassland: Why Artemisia ordosica declines on old fixed sand dunes. Acta Phytoecologica Sinica, 24, 275-279. [郭柯, 董学军, 刘志茂 (2000). 毛乌素沙地沙丘土壤含水量特点— 兼论老固定沙地上油高衰退原因. 植物生态学报, 24 , 275-279.]

Hallett LM, Hsu JS, Cleland EE, Collins SL, Dickson TL, Farrer EC, Gherardi LA, Gross KL, Hobbs RJ, Turnbull L, Suding KN (2014). Biotic mechanisms of community stability shift along a precipitation gradient. Ecology, 95, 1693-1700.

IPCC (2013). Climate Change 2013: The Physical Science Basis. Cambridge University Press, Cambridge, UK. 1535.

Kawamura K, Akiyama T, Yokota HO, Tsutsumi M, Yasuda T, Watanabe O, Wang SP (2005). Quantifying grazing intensities using geographic information systems and satellite remote sensing in the Xilingol steppe region, Inner Mongolia, China. Agriculture, Ecosystems \& Environment, 107, 83-93.

Knapp AK, Beier C, Briske DD, Classen AT, Luo YQ, Reichstein M, Smith MD, Smith SD, Bell JE, Fay PA, Heisler JL, Leavitt SW, Sherry R, Smith B, Wen ES (2008). Consequences of more extreme precipitation regimes for terrestrial ecosystems. BioScience, 58, 811-821.

Knapp AK, Briggs JM, Koelliker JK (2001). Frequency and extent of water limitation to primary production in a mesic temperate grassland. Ecosystems, 4, 19-28.

Knapp AK, Ciais P, Smith MD (2017). Reconciling inconsis- tencies in precipitation-productivity relationships: Implications for climate change. New Phytologist, 214, 41-47.

Knapp AK, Fay PA, Blair JM, Collins SL, Smith MD, Carlisle JD, Harper CW, Danner BT, Lett MS, McCarron JK (2002). Rainfall variability, carbon cycling, and plant species diversity in a mesic grassland. Science, 298, 2202-2205.

Knapp AK, Medina E (1999). Success of $C_{4}$ photosynthesis in the field: Lessons from communities dominated by $\mathrm{C}_{4}$ plants. In: Sage RF, Monson RK eds. $C_{4}$ Plant Biology. Academic Press, New York. 251-283.

Knapp AK, Smith MD (2001). Variation among biomes in temporal dynamics of aboveground primary production. Science, 291, 481-484.

Lauenroth WK, Sala OE (1992). Long-term forage production of north American shortgrass steppe. Ecological Applications, 2, 397-403.

Li ZY, Ma WH, Liang CZ, Liu ZL, Wang W, Wang LX (2015). Long-term vegetation dynamics driven by climatic variations in the Inner Mongolia grassland: Findings from 30-year monitoring. Landscape Ecology, 30, 1701-1711.

Liu B, Chang XX, Li SB (2010). Rainfall patterns and pulse characteristics in desert regions of the Heihe River basin. Acta Ecologica Sinica, 30, 5194-5199. [刘冰, 常学向, 李守波 (2010). 黑河流域荒漠区降水格局及其脉动特 征. 生态学报, 30, 5194-5199.]

Ludwig JA, Tongway DJ, Freudenberger DO, Nobl JC, Hodgkinson KC (1996). Landscape Ecology: Function and Management: Principles from Australia's Rangelands. CSIRO Publishing, Melbourne.

Ma YH, Zhang TH, Liu XP, Mao W, Yue XF (2015). Effects of small rainfall events in spring on germination of Chenopodium acuminatum in Horqin Sandy Land. Acta Ecologica Sinica, 35, 4063-4070. [马望花, 张铜会, 刘新 平, 毛伟, 岳祥飞 (2015). 春季小降雨事件对科尔沁沙 地尖头叶藜萌发的影响. 生态学报, 35, 4063-4070.]

Mowll W, Blumenthal DM, Cherwin K, Smith A, Symstad AJ, Vermeire LT, Collins SL, Smith MD, Knapp AK (2015). Climatic controls of aboveground net primary production in semi-arid grasslands along a latitudinal gradient portend low sensitivity to warming. Oecologia, 177, 959-969.

Nippert JB, Fay PA, Carlisle JD, Knapp AK, Smith MD (2009). Ecophysiological responses of two dominant grasses to altered temperature and precipitation regimes. Acta Oecologica, 35, 400-408.

Niu SL, Jiang GM, Li YG (2004). Environmental regulations of $\mathrm{C}_{3}$ and $\mathrm{C}_{4}$ plants. Acta Ecologica Sinica, 24, 308-314. [牛书丽, 蒋高明, 李永庚 (2004). $\mathrm{C}_{3}$ 与 $\mathrm{C}_{4}$ 植物的环境调 控. 生态学报, 24, 308-314.]

Niu SL, Wu MY, Han Y, Xia JY, Li LH, Wan SQ (2007). Water-mediated responses of ecosystem carbon fluxes to climatic change in a temperate steppe. New Phytologist, 177, 209-219. 
Noy-Meir I (1973). Desert ecosystems: Environment and producers. Annual Review of Ecology and Systematics, 4, $25-51$.

Owens MK, Lyons RK, Alejandro CL (2006). Rainfall partitioning within semiarid juniper communities: Effects of event size and canopy cover. Hydrological Processes, 20, 3179-3189.

Ren ZY, Yan JP, Wang PT (2016). Spatio-temporal variations of precipitation concentration degree and precipitation concentration period in Inner Mongolia. Journal of Desert Research, 36，760-766. [任志艳, 延军平, 王鹏涛 (2016). 1960-2012年内蒙古降水集中度和降水集中期时 空变化. 中国沙漠, 36, 760-766.]

Reynolds JF, Kemp PR, Ogle K, Fernández RJ (2004). Modifying the "pulse-reserve" paradigm for deserts of North America: Precipitation pulses, soil water, and plant responses. Oecologia, 141, 194-210.

Reynolds JF, Kemp PR, Tenhunen JD (2000). Effects of long-term rainfall variability on evapotranspiration and soil water distribution in the Chihuahuan Desert: A modeling analysis. Plant Ecology, 150, 145-159.

Robertson TR, Bell CW, Zak JC, Tissue DT (2009). Precipitation timing and magnitude differentially affect aboveground annual net primary productivity in three perennial species in a Chihuahuan Desert grassland. New Phytologist, 181, 230-242.

Robertson TR, Zak JC, Tissue DT (2010). Precipitation magnitude and timing differentially affect species richness and plant density in the sotol grassland of the Chihuahuan Desert. Oecologia, 162, 185-197.

Sala OE, Lauenroth WK (1982). Small rainfall events: An ecological role in semiarid regions. Oecologia, 53, 301-304.

Sala OE, Parton WJ, Joyce LA, Lauenroth WK (1988). Primary production of the central grassland region of the United States. Ecology, 69, 40-45.

Schwinning S, Sala OE (2004). Hierarchy of responses to resource pulses in arid and semi-arid ecosystems. Oecologia, 141, 211-220.

Schwinning S, Sala OE, Loik ME, Ehleringer JR (2004). Thresholds, memory, and seasonality: Understanding pulse dynamics in arid/semi-arid ecosystems. Oecologia, 141, 191-193.

Sims PL, Singh JS, Lauenroth WK (1978). The structure and function of ten western north American grasslands: I. Abiotic and vegetational characteristics. Journal of Ecology, 66, 251-285.

Snyder KA, Donovan LA, James JJ, Tiller RL, Richards JH (2004). Extensive summer water pulses do not necessarily lead to canopy growth of Great Basin and northern Mojave
Desert shrubs. Oecologia, 141, 325-334.

Soriano A, Sala O (1984). Ecological strategies in a Patagonian arid steppe. Vegetatio, 56, 9-15.

Swemmer AM, Knapp AK, Snyman HA (2007). Intra-seasonal precipitation patterns and above-ground productivity in three perennial grasslands. Journal of Ecology, 95, 780-788.

Tan LP, Zhou GS (2013). Variations of Leymus chinesis community, functional groups, plant species and their relationships with climate factors. Acta Ecologica Sinica, 33, 650-658. [谭丽萍, 周广胜 (2013). 内蒙古羊草群落、功 能群、物种变化及其与气候的关系. 生态学报, 33 , 650-658.]

Tong C, Wu J, Yong S, Yang J, Yong W (2004). A landscapescale assessment of steppe degradation in the Xilin River Basin, Inner Mongolia, China. Journal of Arid Environments, 59, 133-149.

Walter H (1971). Natural savannahs as a transition to the arid zone. In: Walter H ed. Ecology of Tropical and Subtropical Vegetation. Oliver and Boyd, Edinburgh, UK. 238-265.

Wiesmeier M, Steffens M, Kölbl A, Kögel-Knabner I (2009). Degradation and small-scale spatial homogenization of topsoils in intensively-grazed steppes of Northern China. Soil \& Tillage Research, 104, 299-310.

Zhang LJ, Qian YF (2004). A study on the feature of precipitation concentration and its relation to flood-producing in the Yangtze River Valley of China. Chinese Journal of Geophysics, 47, 622-630. [张录军, 钱永甫 (2004). 长江 流域汛期降水集中程度和洪涝关系研究. 地球物理学 报, 47, 622-630.]

Zhao WZ, Liu H (2011). Precipitation pulses and ecosystem responses in arid and semiarid regions: A review. Chinese Journal of Applied Ecology, 22, 243-249. [赵文智, 刘鹄 (2011). 干旱、半干旱环境降水脉动对生态系统的影响. 应用生态学报, 22, 243-249.]

Zhao XY, Liu LX, Wang W, Chang XL, Qu H, Mao W, Jia KF (2014). Impacts of precipitation change on desert-grassland vegetation productivity. Journal of Desert Research, 34, 1486-1495. [赵学勇, 刘良旭, 王玮, 常学礼, 曲浩, 毛 伟, 贾昆峰 (2014). 降水波动对荒漠草原生产力的影 响. 中国沙漠, 34, 1486-1495.]

Zheng XQ, Zheng XJ, Li Y (2012). Distribution and change of different precipitation pulse sizes in the southern marginal zone of the Junggar Basin, China. Arid Zone Research, 29, 495-502. [郑新倩, 郑新军, 李彦 (2012). 准噶尔盆地 南缘降水脉冲量级分布及其变化规律. 干旱区研究, 29 , 495-502.]

责任编委: 唐志尧 责任编辑: 李 敏 实习编辑: 赵 航 